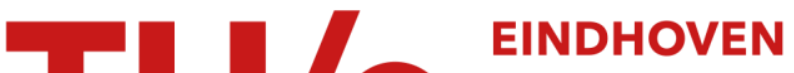

\section{Phase-space manipulation of ultracold ion bunches with time- dependent fields}

\section{Citation for published version (APA):}

Reijnders, M. P., Debernardi, N., Geer, van der, S. B., Mutsaers, P. H. A., Vredenbregt, E. J. D., \& Luiten, O. J. (2010). Phase-space manipulation of ultracold ion bunches with time-dependent fields. Physical Review Letters, 105(3), 034802-1/4. [034802]. https://doi.org/10.1103/PhysRevLett.105.034802

DOI:

10.1103/PhysRevLett.105.034802

Document status and date:

Published: 01/07/2010

\section{Document Version:}

Publisher's PDF, also known as Version of Record (includes final page, issue and volume numbers)

\section{Please check the document version of this publication:}

- A submitted manuscript is the version of the article upon submission and before peer-review. There can be important differences between the submitted version and the official published version of record. People interested in the research are advised to contact the author for the final version of the publication, or visit the $\mathrm{DOI}$ to the publisher's website.

- The final author version and the galley proof are versions of the publication after peer review.

- The final published version features the final layout of the paper including the volume, issue and page numbers.

Link to publication

\section{General rights}

Copyright and moral rights for the publications made accessible in the public portal are retained by the authors and/or other copyright owners and it is a condition of accessing publications that users recognise and abide by the legal requirements associated with these rights.

- Users may download and print one copy of any publication from the public portal for the purpose of private study or research.

- You may not further distribute the material or use it for any profit-making activity or commercial gain

- You may freely distribute the URL identifying the publication in the public portal.

If the publication is distributed under the terms of Article 25fa of the Dutch Copyright Act, indicated by the "Taverne" license above, please follow below link for the End User Agreement:

www.tue.nl/taverne

Take down policy

If you believe that this document breaches copyright please contact us at:

openaccess@tue.nl

providing details and we will investigate your claim. 


\title{
Phase-Space Manipulation of Ultracold Ion Bunches with Time-Dependent Fields
}

\author{
M. P. Reijnders, N. Debernardi, S. B. van der Geer, P. H. A. Mutsaers, E. J. D. Vredenbregt, and O. J. Luiten \\ Department of Applied Physics, Eindhoven University of Technology, P.O. Box 513, 5600 MB Eindhoven, The Netherlands
}

(Received 3 February 2010; published 15 July 2010)

\begin{abstract}
All applications of high brightness ion beams depend on the possibility to precisely manipulate the trajectories of the ions or, more generally, to control their phase-space distribution. We show that the combination of a laser-cooled ion source and time-dependent acceleration fields gives new possibilities to perform precise phase-space control. We demonstrate reduction of the longitudinal energy spread and realization of a lens with control over its focal length and sign, as well as the sign of the spherical aberrations. This creates new possibilities to correct for the spherical and chromatic aberrations which are presently limiting the spatial resolution.
\end{abstract}

DOI: 10.1103/PhysRevLett.105.034802

High brightness ion beams are crucial for a variety of applications such as focused ion beam systems [1] and ion microscopes [2]. Recently, a new kind of ion source was proposed, the ultracold ion source (UCIS) [3,4], based on laser-cooled atoms. The UCIS is characterized by a low effective source temperature [5] and an extended source size [3]. Compared to liquid metal ion sources [6] and ionic liquid ion sources [7], it offers the advantage of a much reduced energy spread [8] and thus reduced chromatic aberration in focused ion beam columns at comparable (predicted) brightness. In comparison with the He-gas field emission source [2], the brightness is lower, but stable operation is possible with isotopically pure and strictly singly ionized beams of several ionic species, both light (e.g., $\mathrm{Li}^{+}$) as well as heavy (e.g., $\mathrm{Rb}^{+}$and $\mathrm{Cs}^{+}$). This makes it an attractive source for ion microscopy as well for sputtering applications. In this Letter, we show that, additionally, the UCIS enables manipulation of the ions by time-dependent electric fields to control both the longitudinal and the transverse phase-space distribution. Not only linear but also higher order beam manipulations are possible, which opens exciting new possibilities to correct for spherical and chromatic aberrations, which are presently limiting the spatial resolution [1].

The idea of time-dependent manipulation has been discussed before. In secondary ion mass spectroscopy $[9,10]$, for example, it is used to improve the mass resolution. Aberration correction with time-dependent electric [11,12] and magnetic fields [13] has been the subject of several theoretical studies but has not yet been demonstrated experimentally [11]. The use of time-dependent fields to manipulate ion bunches is therefore not limited to the UCIS source; however, the properties of the UCIS make practical implementation easier. The low initial thermal velocities and the extended source area of the UCIS allow the creation of well-defined beams at very low energies [8]. The small velocities of the ions then allow temporal manipulation at experimentally feasible time scales. In this Letter, we demonstrate how this may be used for reduction of the longitudinal energy spread caused by the initial size
PACS numbers: 41.85.Gy, 32.80.Fb, 41.75.Ak

of the source, the limiting factor up to now [8]. We also show that it is possible to create a versatile, fully adjustable lens with control over the sign of the focal length and the spherical aberration coefficient. Importantly, we circumvent Scherzer's theorem [11,14], a major restriction in conventional static cylindrical systems, which states that spherical aberration coefficients are always positive, so cancellation of the aberration is not possible. With timedependent fields there is no such restriction. We show that, by switching the lens voltage, the sign of the coefficient can be reversed. This may lead to the development of aberration-corrected focusing columns.

In our UCIS experiments, a laser-cooled atomic cloud is trapped inside a cylindrically symmetric accelerator structure. Part of the cloud is pulsed ionized to create ion bunches, which are subsequently accelerated in the $z$ direction; see Fig. 1. The symmetry of the system makes it possible to write the complete field as an expansion in the $z$ component of a (static) on-axis electric field $E_{z}(z)$ [15]. The time-dependent electric field is modeled as $E_{z}(z, t)=$ $e_{z}(z) \alpha V_{a}(t)$, with $V_{a}(t)$ the time-dependent anode voltage and $e_{z}(z)=E_{z}(z) / \int_{0}^{\infty} E_{z}(z) d z$ the normalized electric field distribution on axis, with $\alpha$ the ratio of the potential

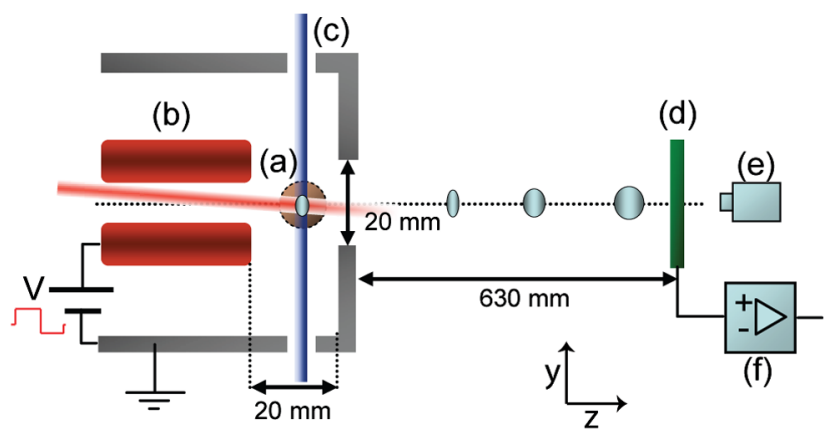

FIG. 1 (color online). Schematic overview of the experiment. A laser-cooled cloud of rubidium (a) is trapped inside an accelerator structure (b). After pulsed ionization (c), ions are accelerated to the detector (d) where the transverse spatial distribution (e) and the temporal distribution are measured (f). 
on the position of the ions and the potential at the cathode. If the bunch size does not change much during acceleration, then the characteristic transverse momentum spread $\Delta p_{r}$ and the characteristic longitudinal momentum spread $\Delta p_{z}$ outside the accelerator field are given by

$$
\begin{aligned}
\Delta p_{r}= & q \alpha \int_{0}^{\infty} V_{a}(t)\left(-\frac{1}{2} e_{z}^{\prime}(z) \sigma_{r_{0}}\right. \\
& \left.+\frac{1}{16} e_{z}^{\prime \prime \prime}(z) \sigma_{r_{0}}^{3}+\cdots\right) d t \\
\Delta p_{z}= & q \alpha \int_{0}^{\infty} V_{a}(t)\left(e_{z}^{\prime}(z) \sigma_{z_{0}}\right. \\
& \left.+\frac{1}{2} e_{z}^{\prime \prime}(z) \sigma_{z_{0}}^{2}+\cdots\right) d t
\end{aligned}
$$

where $q$ is the ion charge, $\sigma_{r_{0}}$ is the initial root mean square (rms) transverse size, $\sigma_{z_{0}}$ is the rms longitudinal size, and a prime denotes the derivative with respect to $z$.

The anode voltage function $V_{a}(t)$ enables independent control of both the linear and higher order terms in the expansion. This is illustrated in Fig. 2(a), where the first term of these expansions is shown as a function of $z$. By switching with a unipolar [Fig. 2(b)] or a bipolar pulse [Fig. 2(c)], the contributions of the different field terms in Eq. (1) can be changed.

Our UCIS consists of a rubidium-85 vapor-cell magneto-optical trap. Typically, $10^{8} \mathrm{Rb}$ atoms are trapped in a cloud with a rms radius of $1 \mathrm{~mm}$. A two-step photoionization process is used to selectively ionize a small volume inside the trapped atomic cloud; see Fig. 1: A focused $780 \mathrm{~nm}$ laser beam propagating along the $z$ direction excites atoms to the $5 p$ level. A $479 \mathrm{~nm}$ laser beam perpendicular to the excitation beam ionizes a fraction of the excited atoms, creating an ion bunch with initial sizes $\sigma_{z_{0}}=200 \pm 20 \mu \mathrm{m}$ and $\sigma_{r_{0}}=250 \pm 30 \mu \mathrm{m}$. For details of the accelerator structure, see [16]. The anode voltage $V_{a}$ can be switched between three states: zero, a positive high voltage level $\left(V_{p}\right)$, and a negative high voltage level $\left(V_{n}\right)$ with a switch time of $50 \mathrm{~ns}$.

At a distance $L=0.64 \mathrm{~m}$ from the center of the accelerator, a $40 \mathrm{~mm}$ diameter multichannel plate detector with phosphor screen is mounted. A CCD camera is used to (a) dc

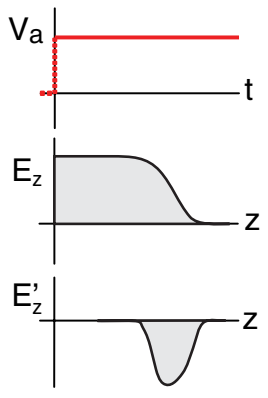

(b) unipolar

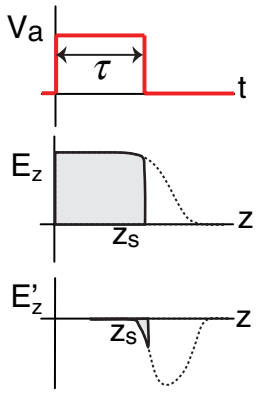

(c) bipolar

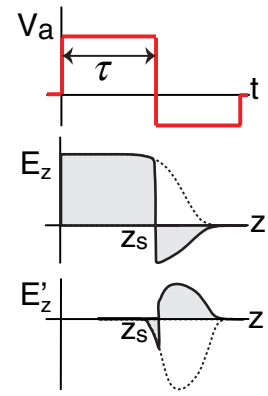

FIG. 2 (color online). Illustration of the field terms in Eqs. (1a) and (1b) for various anode voltage functions $V_{a}(t)$. capture images of the phosphor screen that contain the spatial information of the ion bunches. Simultaneously, the temporal distribution of the ion bunch is recorded on an oscilloscope by using a transimpedance amplifier connected to the phosphor screen. From this the total charge $Q$ of the bunch, the time of flight $T$ to the detector, and its rms spread $\sigma_{T}$ are extracted. The beam energy $U$ is calculated from $T$, with $U=(1 / 2) m(L / T)^{2}$, and the relative energy spread is given by $\sigma_{U} / U=2 \sigma_{T} / T$.

In Fig. 3(a), we present results of the longitudinal phasespace manipulation. By time-of-flight measurements, the beam energy $U$ and the relative energy spread $\sigma_{U} / U$ were determined. The relative energy spread $\sigma_{U} / U$ due to the acceleration can be approximated by $\sigma_{U} / U=2 \Delta p_{z} / p_{z}$, with $p_{z}$ the average ion momentum and $\Delta p_{z}$ given by Eq. (1b). First we look at the dc measurements, indicated by the purple circles in Fig. 3(a). For the dc case $\left[V_{a}(t)=\right.$ constant], Eq. (1b) results to the lowest order in $\sigma_{z_{0}}$ in $\sigma_{U} / U=\sigma_{z_{0}}\left|e_{z}(0)\right|$, with $e_{z}(0)$ the normalized electric field at the initial position of the bunch $(z=0)$. Because $U$ and $\sigma_{U}$ are both proportional to the acceleration voltage, the relative energy spread $\sigma_{U} / U$ is independent of beam energy, as is clearly illustrated by the data in Fig. 3(a). In dc fields, a small relative energy spread can therefore be realized only by choosing a small initial $\sigma_{z_{0}}$. By using time-dependent fields, $\sigma_{U} / U$ can be reduced without changing $\sigma_{z_{0}}$ : With a unipolar switch function of duration $\tau$, the accelerating field can be turned off while the ion bunch is still being accelerated. The time spent in the field

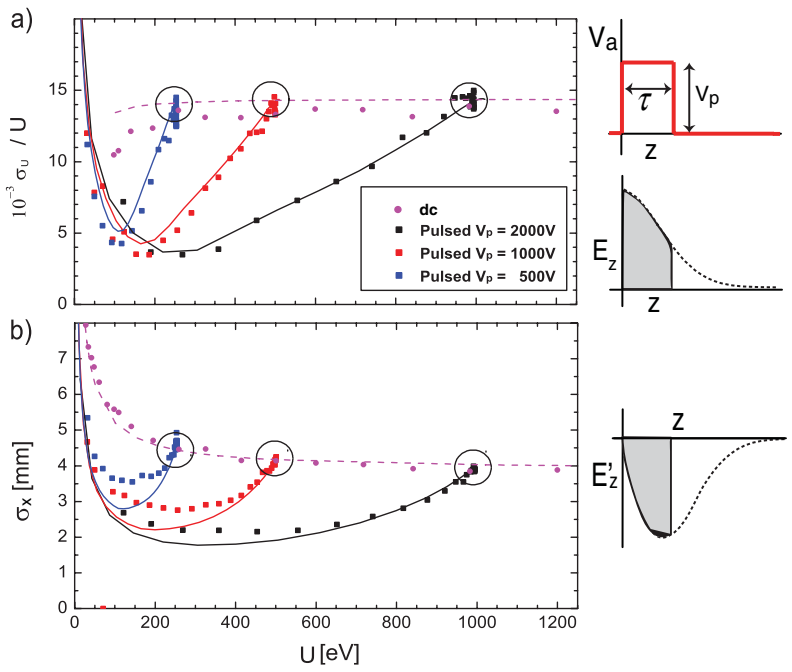

FIG. 3 (color). Results of measurement with a unipolar voltage pulse. The beam energy $U$ is varied by changing the pulse duration $\tau$ from 100 ns up to $2 \mu$ s while keeping $V_{p}$ constant. Three curves (black, red, and blue squares) with, respectively, $V_{p}=1000,500$, and $250 \mathrm{~V}$ are shown. In (a) the longitudinal relative energy spread $\sigma_{U} / U$ versus $U$ is plotted and in (b) the transverse size on the detector $\sigma_{x}$ in the $x$ direction versus $U$. Additionally, a dc measurement (purple circles) is presented where $V_{p}$ is varied. Particle tracking simulations are depicted as solid curves (unipolar pulses) and a dashed curve (dc). 
is then the same for all ions, independent of their initial position. From Eq. (1b) then follows

$$
\frac{\sigma_{U}}{U}=\sigma_{z_{0}}\left|e_{z}\left(z_{s}\right)-e_{z}(0)\right| \text {, }
$$

with $z_{s}$ the position of the center of the bunch when the field is turned off $(t=\tau)$. If the field is switched off after the ions have left the accelerator field, Eq. (2) reduces to the expression derived for the dc case. In the idealized case of a perfectly homogeneous electric field inside the accelerator, i.e., $e_{z}\left(z_{s}\right)=e_{z}(0)$, the first term exactly cancels the second term in Eq. (2). If the field is not homogeneous, the first term still partially reduces the energy spread.

In Fig. 3(a), measurements are shown performed with such a unipolar pulse. The relative energy spread $\sigma_{U} / U$ is measured as a function of $U$ by varying $\tau$ from $100 \mathrm{~ns}$ up to $2 \mu \mathrm{s}$ for three different $V_{p}=2000,1000$, and $500 \mathrm{~V}$ at a bunch charge of $\approx 0.5 \mathrm{fC}$. The encircled points correspond to pulse durations for which the ions have already left the accelerator at $t=\tau$ and are therefore not influenced by the field switching. In that case $\sigma_{U} / U$ coincides with the dc measurements. For shorter $\tau$, both $U$ and $\sigma_{U}$ are reduced, but $\sigma_{U}$ reduces more than $U$, because the influence of the field derivatives in Eq. (1b) is smaller. For even shorter $\tau, T$ becomes so long that space-charge effects start to increase $\sigma_{U}$. This is the same effect as observed in Ref. [8]. In the region where space-charge effects are not important, the curves for different $V_{p}$ have the same shape and can in fact be made to overlap by scaling the $U$ axis proportional to $V_{p}$.

To quantitatively understand the measurements, particle tracking simulations were performed with the GPT [17] code. All the ions in the bunch are tracked individually with all mutual Coulomb interactions included. The initial conditions are the same for all simulations and are chosen with values within measurement uncertainty. The simulation results are depicted in Fig. 3. Good agreement with the data is obtained. Small deviations from the simulations occur in the low-energy region, where space-charge effects become significant. A reduction of a factor of 3 of the relative energy spread is obtained. To improve the reduction further, optimization of the field shape is required.

Now we discuss manipulation of the transverse phase space. Simultaneously with the time-of-flight signals, the spatial ion bunch distribution on the detector was captured by the CCD camera. This is shown in Fig. 3(b), where the transverse spot size $\sigma_{x}$ on the detector is plotted as a function of $U$. The behavior in the longitudinal $(z)$ and the transverse $(x)$ direction is very similar, as expected in view of the strong similarities between Eqs. (1a) and (1b).

The divergent field at the exit hole of the accelerator structure acts as a lens with a focal strength given by $1 / f=$ $-\Delta p_{r} /\left(\sigma_{r_{0}} p_{z}\right)$. The lens effect is described by the first term in the expansion in Eq. (1a). When the bunch velocity is assumed constant while passing through the divergent part of the field, a simple expression can be derived. In the dc case this results in $1 / f=-1 / 4 e_{z}(0)$, corresponding to a negative focal length $f=-33 \mathrm{~mm}$ for our accelerator structure. This lens effect can be observed in the dc measurements in Fig. 3(b). At high energy; the bunch size is increased due to the lens by a constant factor of $16 \mathrm{com}-$ pared to the initial size; at lower energies $(U<200 \mathrm{eV})$, the spot blows up even further due to space charge.

The results of the unipolar experiments are indicated by the black, red, and blue symbols in Fig. 3(b). For this unipolar case, the focal strength can be written as

$$
\frac{1}{f}=\frac{1}{4}\left[e_{z}\left(z_{s}\right)-e_{z}(0)\right]
$$

The encircled points of the curves correspond to measurements where $\tau$ is again so long that the ions experience the full divergent field. When $\tau$ is reduced, the field is switched off while the ions are still in a divergent part of the field, so the radial momentum spread $\Delta p_{r}$ is reduced [see the field diagram in Fig. 3(b)]. As is clear from Eq. (3), the negative lens strength is then also reduced, resulting in a smaller spot on the screen. At even shorter $\tau$, and thus lower $U$, space charge starts to increase the spot size again.

Instead of simply turning the field off with a unipolar pulse, it is also possible to change the sign of the radial electric field with a bipolar pulse (see Fig. 4). By choosing a suitable bipolar pulse, the radial momentum an ion receives can be inverted, so the diverging accelerator field can now be used as a positive lens. For this pulse the focal strength can be approximated with

$$
\frac{1}{f}=\frac{1}{4}\left(\frac{V_{p}-V_{n}}{V_{p}} e_{z}\left(z_{s}\right)-e_{z}(0)\right) .
$$

Clearly, the focal strength can be controlled by changing the duration and amplitudes of the pulse. As a side effect, also the field in the $z$ direction is reversed, so the ions start to decelerate.

In Fig. 4, measurements are shown of the spot size on the detector when a bipolar pulse with $V_{p}=1000 \mathrm{~V}$ and

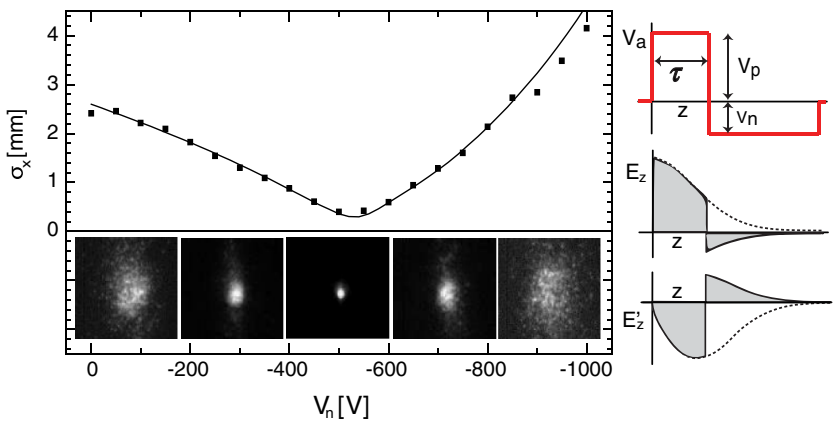

FIG. 4 (color online). Demonstration of focusing by bipolar voltage pulses with $V_{p}=1000 \mathrm{~V}$. The transverse size in the $x$ direction $\sigma_{x}$ of the bunch on the detector is measured as a function of $V_{n}$. Phosphor images of the beam are shown at the bottom. The solid curve corresponds to particle tracking simulations. 


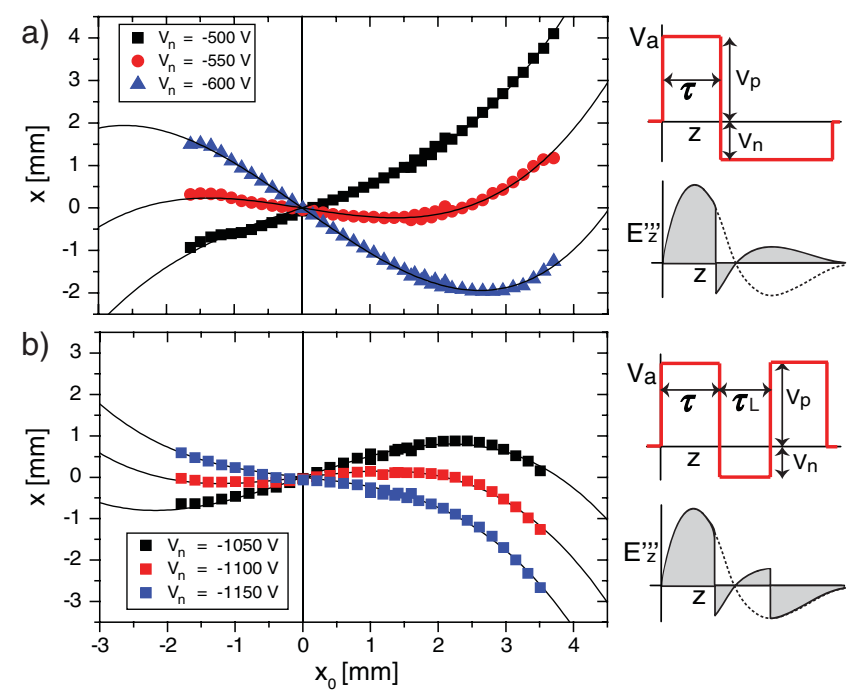

FIG. 5 (color). Demonstration of the sign reversal of the spherical aberration coefficients. The position $x$ of the ion bunch on the detector is recorded as a function of the initial ionization volume position $x_{0}$ (scatter plots). In all measurements $V_{p}=$ $1000 \mathrm{~V}$. The curves are fitted with the relation $x=A x_{0}-C x_{0}^{3}$ (solid curves). Results are shown of measurements using (a) a bipolar pulse $(C<0)$ and (b) a tripolar pulse $(C>0)$.

$\tau=633 \mathrm{~ns}$ is applied. The negative voltage $V_{n}$ has a duration longer than the time the bunch spends in the accelerator. The figure clearly shows that, when $V_{n}$ is increased, the bunch first focuses on the detector and then starts to overfocus. Phosphor screen images are added to illustrate the effect. The bunch is focused on the detector when $V_{n}=540 \mathrm{~V}$; in that case it is accelerated to $U=280 \mathrm{eV}$ with the positive pulse and decelerated to $U=180 \mathrm{eV}$ by the negative pulse. Again, measurements agree well with particle tracking simulations, indicated by the solid curve.

So far, we have shown that we can control the linear term in Eqs. (1a) and (1b) with the time-dependent fields, but control of higher orders is also possible. As a demonstration, we change the spherical aberration due to the exit fields of the accelerator. To determine the amount of spherical aberration, the transverse position $x$ on the detector is measured as a function of the initial position $x_{0}$. The position $x_{0}$ is changed by moving the ionization laser focus. When the exit lens is aberration-free, the relation between $x_{0}$ and $x$ is linear $\left(x=A x_{0}\right)$; when spherical aberrations are present, a third-order term appears $(x=$ $\left.A x_{0}-C x_{0}^{3}\right)$, which is the result of the $e_{z}^{\prime \prime \prime}$ term in Eq. (1a). By changing $V_{a}(t)$, the integral of this term can be controlled (Fig. 5). From the coefficient $A$, the focal length is obtained by $f=-L /(A-1)$ and the third-order coefficient $C$ is related to the $C_{s}$ coefficient by $C_{s}=f^{3} C$, if the bunch is focused on the detector. The spot size $\delta_{s}$ due to spherical aberrations in a focusing system is given by $\delta_{s}=C_{s} \alpha^{3}$, with $\alpha$ the lens acceptance angle [11].
A bipolar and a tripolar switch function were used in the experiment in Fig. 5. For each, three measurements are shown with $f \simeq L$. A horizontal line $(A=0)$ would correspond to a focus precisely on the detector, a positive linear slope to a focus behind the detector, and a negative slope to a focus in front of the detector. In Fig. 5(a), the deviation from linear behavior is clear and fits well with a third-order term (solid curves). The bipolar pulse results in $C_{s}=$ $-1.1 \pm 0.1 \times 10^{4} \mathrm{~m}$. By changing only to a tripolar pulse [Fig. 5(b)], the sign of the aberration coefficient is reversed to $C_{s}=4.0 \pm 0.2 \times 10^{4} \mathrm{~m}$. This shows that manipulation with time-dependent fields can be used to achieve aberration-corrected systems.

In conclusion, we have shown that phase-space manipulation of ultracold ion beams with time-dependent acceleration fields is experimentally feasible. We have shown that a unipolar pulse can be used to reduce the relative energy spread, a bipolar pulse can be used to change the sign of a lens, and, finally, a tripolar pulse can be used to change the sign of the spherical aberration coefficient. Even more complex pulses will enable further possibilities.

This research is supported by the Dutch Technology Foundation STW, applied science division of NWO, and the Technology Program of the Ministry of Economic Affairs.

[1] J. Orloff, L. Swanson, and M. Utlaut, High Resolution Focused Ion Beams FIB and Applications (Academic, New York, 2002).

[2] B. W. Ward, J. A. Notte, and N. P. Economou, J. Vac. Sci. Technol. B 24, 2871 (2006).

[3] S. B. van der Geer et al., J. Appl. Phys. 102, 094312 (2007).

[4] J. L. Hanssen, J. J. McClelland, E. A. Dakin, and M. Jacka, Phys. Rev. A 74, 063416 (2006).

[5] J. L. Hanssen, S. B. Hill, J. Orloff, and J. J. McClelland, Nano Lett. 8, 2844 (2008).

[6] Handbook of Charged Particle Optics, edited by J. Orloff (CRC Press, Boca Raton, 1997).

[7] A. N. Zorzos and P. C. Lozano, J. Vac. Sci. Technol. B 26, 2097 (2008).

[8] M. P. Reijnders et al., Phys. Rev. Lett. 102, 034802 (2009).

[9] W.C. Wiley and I. H. McLaren, Rev. Sci. Instrum. 26, $1150(1955)$

[10] S. V. Kovtoun, R. D. English, and R. J. Cotter, J. Am. Soc. Mass Spectrom. 13, 135 (2002).

[11] P. W. Hawkes, Phil. Trans. R. Soc. A 367, 3637 (2009).

[12] G. Schönhense and H. Spiecker, J. Vac. Sci. Technol. B 20, 2526 (2002)

[13] M. Calvo, Ultramicroscopy 99, 179 (2004).

[14] O. Scherzer, Z. Phys. 101, 593 (1936).

[15] P. W. Hawkes and E. Kasper, Principles of Electron Optics (Kluwer Academic, Dordrecht, 1989), Vol. 1.

[16] G. Taban et al., Phys. Rev. ST Accel. Beams 11, 050102 (2008).

[17] http://www.pulsar.nl/gpt. 